\title{
Gut microbial composition in different castes and developmental stages of the invasive hornet Vespa velutina nigrithorax
}

\author{
Alessandro Cini ${ }^{\mathrm{b}, *, 1}$, Niccolò Meriggi ${ }^{\mathrm{a}, 1}$, Giovanni Bacci ${ }^{\mathrm{a}}$, Federico Cappa ${ }^{\mathrm{a}}$, Francesco Vitali ${ }^{\mathrm{a}, \mathrm{c}}$, \\ Duccio Cavalieri ${ }^{\mathrm{a}, * *}$, Rita Cervo ${ }^{\mathrm{a}}$ \\ a Department of Biology, University of Florence, Via Madonna del Piano, 50019 Firenze, Italy \\ b Centre for Biodiversity and Environment Research, University College London, Gower Street, London WC1E 6BT, UK \\ c Institute of Biology and Agrarian Biotechnology, National Research Council (IBBA-CNR), Via Moruzzi 1, 56124 Pisa, Italy
}

\section{H I G H L I G H T S}

- Vespa velutina is an alien hornet of high ecological and economic concern in Europe.

- Its gut microbiome has been characterized through meta-genomics.

- We investigated the effect of ontogeny, phenotype and similarity with environment.

- Fungal and bacterial community's abundance depends on ontogeny and phenotype.

- Microbiome characterization is a springboard for management strategies.

\section{A R T I C L E I N F O}

\section{Article history:}

Received 19 March 2020

Received in revised form 8 July 2020

Accepted 8 July 2020

Available online 15 July 2020

Editor: Lotfi Aleya

\section{Keywords:}

Gut microbiome

Vespidae

Social insects

Alien species

Yeasts

Bacteria

\section{G R A P H I C A L A B S T R A C T}
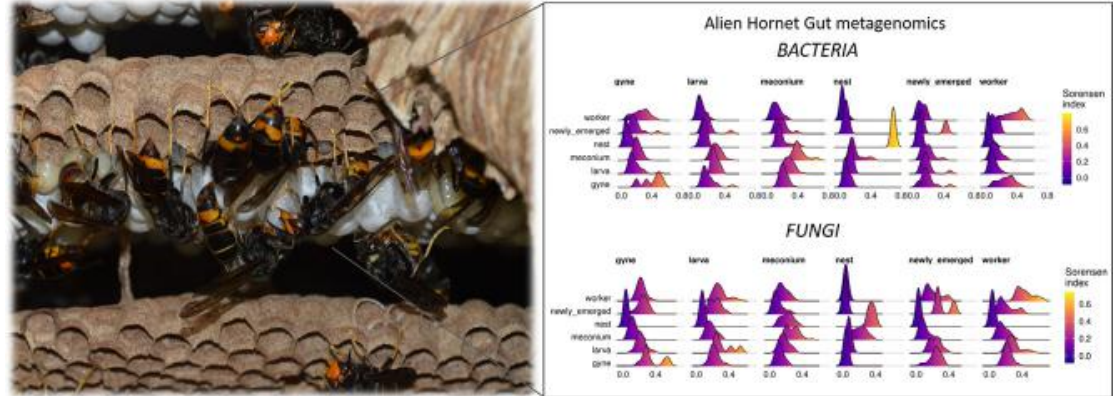

\begin{abstract}
A B S T R A C T
Social insects are successful animal invaders. Their survival and success, and in some cases also their impact on invaded ecosystem functioning, is often mediated by symbiosis with microorganisms. Here, we report a comprehensive comparative characterization of the gut microbial communities of different castes and developmental stages of the invasive hornet Vespa velutina nigrithorax. The species recently colonized Europe, becoming a high ecological and economic concern, as it threatens pollinator survival and competes with native hornet species. We used targeted meta-genomics to describe the yeasts and bacteria gut communities of individuals of different reproductive phenotypes (workers and future queens), life stages (larvae, newly emerged individuals and adults) and colony non-living samples (nest paper and larval faeces). Bacilli, Gammaproteobacteria, Actinobacteria, Alphaproteobacteria were the most abundant classes of bacteria, and Saccharomycetes, Dothideomycetes, Tremellomycetes and Eurotiomycetes were the most represented yeast classes. We found that the microbial compositions significantly differ across developmental stages and castes, with yeast and bacterial communities switching in frequency and abundance during ontogeny and according to reproductive phenotype. Moreover, the gut microbial communities poorly mirror those found in the nest, suggesting that hornets possess a specific microbial signature. Our results provide the first metagenomic resource of the microbiome of $V$. velutina in Europe and suggest the importance of considering life stages, reproductive phenotypes and nest influence in order to obtain a comprehensive picture of social insect microbial communities.
\end{abstract}

(c) 2020 Elsevier B.V. All rights reserved.

\footnotetext{
* Correspondence to: A. Cini, Department of Biology, University of Florence, Via Madonna del Piano, 50019 Firenze, Italy.

** Corresponding author.

E-mail addresses: cini.ales@gmail.com (A. Cini), cavalieri.unifi@gmail.com (D. Cavalieri).

1 Alessandro Cini and Niccolò Meriggi equally contributed to this work.
} 


\section{Introduction}

A major feature of the current 'Anthropocene' epoch is the fast and increasing loss of biodiversity, which affects the functioning of natural ecosystems and threatens human well-being. Insects are among the animal taxa that are suffering the most marked decrease in their abundance and diversity: a recent study estimated a loss of $75 \%$ of the biomass of flying insects in protected areas in Germany over the last 30 years (Leather, 2018). Such a huge loss is especially worrying when regarding important ecosystem services' providers such as social insects. Many species of bees, wasps, ants and termites are involved in pollination, predation, seed dispersal and nutrient recycling to such an extent that they are often considered among "the little things that run the world" (Wilson, 1987; Del Toro et al., 2012). In addition to providing essential ecosystem services, some social insect species also cause severe negative impacts on the environment as well as on human activities, as in the case of accidental species translocation from native to new habitats (i.e. biological invasions) (Chapman and Bourke, 2001). The changes in social insect diversity, abundance and community composition is likely mirrored by changes in ecosystem functioning, potentially leading to what has been defined as an "Ecological Armageddon" (Leather, 2018; but see also Wagner, 2019; Cardoso and Leather, 2019; Outhwaite et al., 2020).

The interaction between social insects and their environment, and consequently their impact on it, can be mediated by symbiosis with microorganisms (Zientz et al., 2005; Hughes et al., 2008). Social insects host a wide range of microorganisms, with a particularly rich community inhabiting the intestinal tract. Gut microbial communities can strongly influence their survival and fitness by mediating crucial life history traits such as nutritional ecology, immunity and reproduction (Koch and Schmid-Hempel, 2011; Mutinelli, 2011; Rosengaus et al., 2011; Engel and Moran, 2013; Meriggi et al., 2019). Moreover, symbiosis with gut microorganisms underlies some of the most astonishing and important functions, namely cellulose digestion from wood fibres in termites as well as nitrogen fixation in herbivorous ants (Radek, 1999; Pinto-Tomás et al., 2009; Suen et al., 2010; Brune and Dietrich, 2015). Furthermore, social insects may also positively affect reproduction, survival and dispersal of gut microorganisms, as it has been suggested for the economically significant Saccharomyces yeasts which inhabit social wasps guts (Stefanini et al., 2012; Dapporto et al., 2016).

Understanding the diversity and composition of gut microbial communities, as well as how they change and interact with their social insect host features, is therefore of overwhelming importance to understand the interaction between social insects and their environment. There is also potential to use this knowledge to manage the positive and negative impacts of social insects on ecosystems and human activities. For example, the understanding of honeybee gut microbiota is a promising tool to improve its health, thus securing and increasing its pollination services (Anderson and Ricigliano, 2017). On the other hand, the manipulation of the microbiota in pest and invasive species may result in significant practical applications for the development of management strategies (Microbial Resource Management, MRM) (Douglas, 2007; Crotti et al., 2012).

To exploit gut microbial communities in a MRM approach, a first crucial step is to undertake their description in terms of diversity and functionality (Crotti et al., 2012). Despite the growing body of knowledge on social insect gut microbiota that has been gathered during the last decades, the variability and functions of social insects' microbial communities still suffer from some significant limitations. First, the taxonomic coverage is limited, with most knowledge focused on model organisms or a few particularly economically relevant species (taxonomic limitation). In fact, while more than 15,000 species of social insects have been described, detailed study on the gut microbiota have been carried out only for a handful of species (mainly the honeybee Apis mellifera, and few species of ants and termites)(Engel and Moran, 2013). Current studies suffer also from poor phenotypic coverage (phenotypic limitation); for instance, only a few studies examined in detail the intraspecific variation in microbial communities by sampling many different phenotypes (such as different morphs, or different castes) and/or investigating the dynamic changes through individual life (Berlanga et al., 2011; Diouf et al., 2015; Kapheim et al., 2015; Otani et al., 2019). Finally, studies usually focus on either the bacterial or the yeast communities, rarely describing both at the same time.

Here, we provide a comprehensive characterization of the gut microbial communities (yeast and bacteria) of a social insect of recent economic and ecological interest, the invasive yellow-legged hornet Vespa velutina Lepetier 1836 (hereafter Vv), with a detailed sampling which considered intraspecific phenotypic variability and ontogenetic dynamics. Vv is an invasive hornet species native of South East Asia (Monceau et al., 2014; Monceau and Thiéry, 2017). Its presence (specifically the subspecies $V$. velutina nigrithorax DuBuysson 1905) was first recorded in the South of France, in 2004 and has since then rapidly spread across most Europe (Villemant et al., 2011a, 2011b; Robinet et al., 2018; Granato et al., 2019). Vv has significant ecological, economic and sanitary impacts, leading to it being listed among the invasive alien species of most concern for Europe (Haxaire and Villemant, 2010; Requier et al., 2019; COMMISSION IMPLEMENTING REGULATION (EU) 2016/1141, 2016). The species' ecological impact is due to its heavy predation on a vast array of insect species (Villemant et al., 2011b) and competition with native species sharing a similar ecological niche (Cini et al., 2018). Economically, the main threat posed by Vv is on beekeeping activities, as the yellow-legged hornet is a specialized predator of honeybees (Monceau et al., 2014). Finally, Vv might also have an impact on human health since envenomation of $\mathrm{Vv}$ can induce severe allergic or toxic reactions, resulting in organ failure and death (Chugo et al., 2015; Liu et al., 2015).

The great concern due to Vv arrival, diffusion and impact in Europe, and other invaded countries (Korea, Japan), has boosted research on life-history traits, ecology and behaviour of the species. Currently, however, there is almost no information about gut microbial composition for hornet species in general, and $\mathrm{Vv}$ in particular. The only study so far carried out on $\mathrm{Vv}$ has been performed in another invaded region (Korea) and it investigated gut bacterial communities, but not yeast (Kim et al., 2018).

By using a metataxonomic approach, we aim to address three main research questions concerning the gut microbial composition of $\mathrm{Vv}$. Firstly, we provide a comprehensive characterization of bacterial and yeast gut communities of this species at the front of the invasion in the newly introduced range (Europe), evaluating its richness and diversity. Secondly, we explore to what extent the gut microbiota is conserved, or not, across life stages and across different reproductive phenotypes. Finally, we are interested in understanding how much the species' core microbiota is shared with the nest, which is one of the most likely environmental sources of microbial communities for a hornet. These three research questions are instrumental in providing information of generic importance (i.e. the first characterization of the gut microbiota of this invasive pest species and the understanding of the dynamics of insect gut microbiota across life stage and phenotypes and its resemblance with the nest) as well as opening potential future avenues of research for applied perspectives on this species.

\section{Materials and methods}

\subsection{The model species V. velutina: life-history and colonial cycle}

Vv is a social species with an annual colony cycle (Spradbery, 1973; Matsuura and Yamane, 1990; Monceau et al., 2014). New colonies are founded in spring by single mated queens that survived the wintering diapause (Monceau et al., 2014). By early summer workers start to emerge and take over the duties of rearing the immature brood and providing for the growing colony. Adult workers catch and manipulate 
preys and provide them to developing larvae, which, in exchange, regurgitate drops of liquid, rich in semi-digested nutrients (amino acids), for the colony adults. Before reaching the pupal stage, larvae expel the entire content of their digestive tract which remains at the bottom of the nest cell (meconium). At the end of summer, the colony reaches its peak in terms of number of individuals and the new generations of sexuals (males and reproductive females, i.e. gynes) are produced. After emergence reproductive individuals stay in the nest, where interactions exchange frequently occurs also among adults; mating takes place in autumn, after that, only mated future queens will survive and enter hibernation (Monceau et al., 2014). As other Vespidae species, $\mathrm{Vv}$ build their nest combs with paper material made by collecting plant fibres in the surrounding environment and then by mixing this plant material with saliva (Matsuura and Yamane, 1990; Monceau et al., 2014).

\subsection{Hornets sampling}

Vv combs with sealed brood were collected during October and November 2016 in the surroundings of Ventimiglia (Liguria, Italy), from 4 nests that were gathered by local beekeepers (coordinates: N1: $43^{\circ} 49^{\prime}$ $00^{\prime \prime} \mathrm{N} 7^{\circ} 46^{\prime} 00^{\prime \prime} \mathrm{E}$; N2: $43^{\circ} 51^{\prime} 44^{\prime \prime} \mathrm{N} 7^{\circ} 51^{\prime} 14^{\prime \prime} \mathrm{E}$; N3: 43 $53^{\prime} 01^{\prime \prime} \mathrm{N} 7^{\circ} 50^{\prime} 73^{\prime \prime}$ $\mathrm{E}$; N4: $\left.43^{\circ} 51^{\prime} 21^{\prime \prime} \mathrm{N} 7^{\circ} 43^{\prime} 34^{\prime \prime} \mathrm{E}\right)$. We collected the following samples from each nest: nest paper (a piece of approximately $1 \mathrm{~cm}^{2}$, from the wall of a cell randomly chosen); meconium, i.e. the waste products of larvae, randomly chosen from a cell; larvae (L4-L5 instars); newly emerged females. To be sure that newly emerged females did not have the chance to exchange liquids with other individual or get "contaminated" in any other way, we collected individuals which were ready to emerge from caped cocoons, easily recognized by their exoskeleton consistence and color, and by the fact that they were moving. Moreover, we collected adult gynes and workers. Gynes were sampled directly from the nest combs. We first considered as gynes the biggest females on the comb which, during sampling, did not defend the comb but rather absconded behind it. Their reproductive phenotype was then confirmed during dissection by looking at the abundance and color of fat bodies: as gynes have to overwinter while workers do not, fat storage allows distinguishing between workers (no or almost absent fat storage) and gynes (abundant fat storage) (Cini et al., 2018; Cappa et al., 2019). Workers were sampled at apiaries, in order to be sure about their foraging phenotype (i.e. while they were foraging by preying on bees). Workers were sampled in Octo-

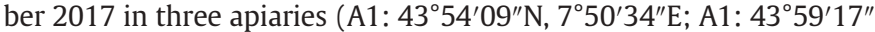
$\mathrm{N}, 7^{\circ} 47^{\prime} 34^{\prime \prime} \mathrm{E}$; A3: $43^{\circ} 49^{\prime} 24^{\prime \prime} \mathrm{N}, 7^{\circ} 44^{\prime} 24^{\prime \prime} \mathrm{E}$ ) in the same area where colonies were collected. All collected samples were immediately stored in RNAlater solution (Invitrogen - Thermo Fisher Scientific) at $-20{ }^{\circ} \mathrm{C}$ until DNA extraction. In order to be protected by hornet stings we wore specific hornet protective suits (EDILGRAPPA Commerciale S.r.l.) as normal beekeeper suits are not enough to protect from stings.

We gathered 32 samples in total, distributed as it follows: 8 from workers, 5 from adult gynes, 3 from newly emerged females, 4 from larvae, and 12 non-insect samples ( 9 meconium samples and 3 nest paper samples). All categories had samples from at least two different nests/ apiaries (details in Supplementary Material, file bacteria_samples.csv and fungi_sample.csv) (Fig. 1).

\subsection{DNA extraction and rRNA genes sequencing}

The insect surface was sterilized with $70 \%$ ethanol. All insect samples were dissected and the guts (midgut and hindgut for adults, whole intestinal sac in larvae) were collected in RNAlater storage and stabilization solution (Invitrogen - Thermo Fisher Scientific) in sterile microcentrifuges tubes and stored at $-20{ }^{\circ} \mathrm{C}$ until DNA extraction. Meconium and nest paper samples were collected and stored using the same protocol. Microbial DNA extraction from all samples was performed using DNeasy PowerLyzer PowerSoil Kit (QIAGEN) following the manufacturer's protocol. Total genomic DNA was then quantified by Tecan Quantification Device (Life Sciences). DNA sequencing was carried out by MiSeq - Illumina platform at BMR Genomics (BMR Genomics sequencing service of University of Padova, Italy, https://www.bmrgenomics.it/). For the study of Bacteria communities, DNA sequencing was carried out on V3-V4 region of the 16S rRNA. V3-V4 region of the 16S rRNA genes was amplified using the primers Pro341F: $5^{\prime}$-CCTACG GGNBGCASCAG-3' and Pro805R: 5'-GACTACNVGGGTATCTAATCC-3' (Takahashi et al., 2014). For the study of Fungi communities, sequencing was carried out targeting the second internal transcribed spacer (ITS2 region). In this case amplification was performed using the primers ITS3: 5' - GCATCGATGAAGAACGCAGC-3' and ITS4: 5'- TCCTCCGCTTA TTGATATGC-3' (White et al., 1990).

\subsection{Sequences pre-treatment and OTUs picking}

Overall sample quality was evaluated with FastQC program (Andrews, 2010). For raw reads quality filtering, primers and other Illumina adapters were removed with Cutadapt (Martin, 2011), and low-quality end of forward and reverse reads were trimmed using Sickle (Joshi and Fass, 2011; quality cut-off of 20 and a length threshold after trimming of 200). MICCA pipeline (ver. 1.7.2, Albanese et al., 2015) was used for OTUs picking as follows: forward and reverse reads were joined with "micamergepairs" command and default parameters, reads with $\mathrm{N}$ bases were discarded with command micca filter. OTUs picking, denoising, and chimera checking was performed with "micca otu" command and using the UNOISE3 protocol (Edgar, 2016) as picking/ denoising algorithm. UNOISE3 performs exact amplicon sequence variants (ASV) reconstruction based on single sequences abundance (i.e. for each sequence, if the abundance is above a threshold the sequence represents a new ASV, if not it is joined with the nearest ASV). In this text, we will use the "OTU" word as synonym of ASV, not with the meaning of similarity-based OTUs but with the meaning of the smaller taxonomic unit considered. Taxonomy was assigned to the representative sequences of the identified OTUs using the RDP classifier and database (ver 2.11, Wang et al., 2007) for 16S rRNA sequences, and using the RDP classifier and the UNITE database (Nilsson et al., 2018) for ITS sequences.

\subsection{Statistical analysis}

All statistical analyses were performed into R environment (version 3.4.4, R Core Team, 2017). Ordinational analyses were computed using Principal Coordinate Analysis based on Bray-Curtis distance with "ordinate" function (phyloseq package version 1.23). Singletons, namely OTUs counting to one in all the dataset, were removed before distance calculation to dampen the hypothesis that extremely rare species may impact the overall distribution of samples. After singleton removal, counts were scaled using DESeq2 package (Love et al., 2014) to account for different sample depth across all samples ('counts' function with 'norm = TRUE'). Scaled counts were used for Bray-Curtis calculation whereas binary data (presence/absence matrix) were used for Sorensen index calculation. Ordinations were analysed using Permutational Multivariate Analysis of Variance (PERMANOVA) using distance matrices as implemented in the "adonis2" function (vegan package version 2.5). Nest origin was used as blocking factor far PERMANOVA analysis to inspect whether different environments had a different microbiome distribution while controlling for the effect of nest. Variation in diversity and composition between sample groups were also assessed using by PERMANOVA pairwise. Beta diversity between developmental stages was computed using the Sorensen index as $S=(B+C) /$ $(2 \mathrm{~A}+\mathrm{B}+\mathrm{C})$, with $\mathrm{A}=$ shared species among groups, $\mathrm{B}=$ species from group $B$ and $C=$ species from groups $C$ ("betadiver" function of vegan package 2.5 ). Beta diversity indices were calculated using the veg dist function (vegan package 2.5). The influence of phenotypes on 


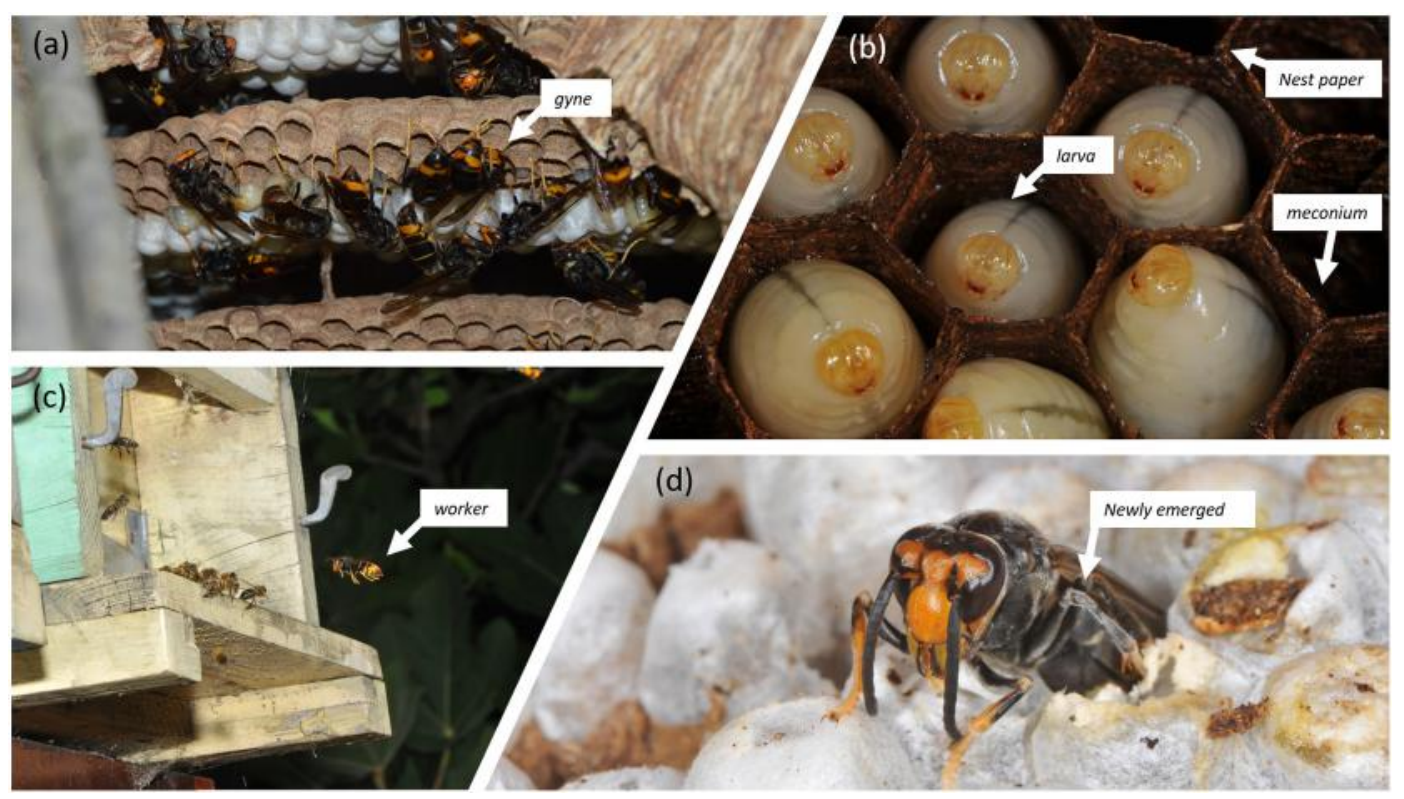

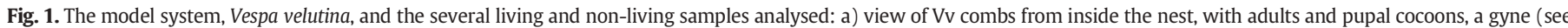

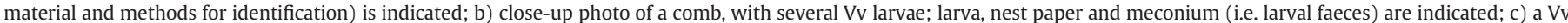
worker is foraging in front of a honey bee hive; d) a newly emerged Vv (photo credits: a) Laura Bortolotti; b), c) and d) Antonio Felicioli).

alpha diversity was inspected thorough one-way analysis of variance (ANOVA) as implemented in the "aov" function of stats native R package. Different alpha diversity indexes were tested: richness (expressed as the number of OTUs detected in a sample), Inverse Simpson index (expressed as 1/D, where D is the Simpson index (Simpson, 1949), and Sorensen index (Shannon, 1948). Species evenness was also calculated and tested using the Pielou's formulation: $H / \log (R)$; where $H$ is the Shannon diversity index and $\mathrm{R}$ is the richness (Pielou, 1966). All alpha diversity indexes were computed using the vegan package, version 2.5 (Oksanen et al., 2019). Differentially abundance OTUs detected assessed using DESeq2 (Love et al., 2014). The influence of sample type was assessed using likelihood ratio test (LRT, "deseq" function) and pvalues were adjusted using the Benjamini and Hochberg approach (also known as "false discovery rate") to reduce type I errors. Colony or apiary of origin was included in the analyses as "origin".

\section{Results}

\subsection{Microbial community composition}

After quality filtering, a total of 2,609,129 16S rRNA and 953,185 ITS reads were obtained, ranging from 3055 to 143,037 and from 799 to 154,965 in all samples, respectively. The number of reads reported a standard deviation of 30,901.17 (for 16S rRNA) and of 37,901.26 (for ITS). A summary of the number of pre-filtering, post-filtering reads for each sample type is reported in supplementary file 1. After singleton removal a total of 2655 OTUs from 16S dataset and 999 OTUs from ITS dataset were obtained (details were reported in supplementary file 1).

The bacterial taxa detected in all samples corresponded to the following main phyla by the order of rank abundance: Firmicutes, Proteobacteria, Actinobacteria and Bacteroidetes. At the class-level the main taxa were represented by Bacilli, Gammaproteobacteria, Actinobacteria, Alphaproteobacteria (Fig. 2f). The fungal phyla detected in all samples corresponded to Ascomycota, Basidiomycota and Zygomycota. The main related class was Saccharomycetes, Dothideomycetes, Tremellomycetes and Eurotiomycetes (Fig. 3f).

\subsection{Gut microbiota shapes across life stages and phenotypes}

Sample type (life stage samples, as well as non-insect samples) significantly shaped the community structure for both bacteria and fungi. PERMANOVA using distance matrices was statistically significant for the sample type $\left(\mathrm{p}<0.001\right.$ and $\mathrm{R}^{2}$ statistic indices 0.45 and 0.4 respectively for bacteria and fungi; Table 1 ). Thus, the analysis showed that the sample type influenced the community structure, and this occurred for each developmental stage and for the non-insect samples (meconium and nest paper).

In accordance with PERMANOVA, Principal Coordinate Analysis (PCoA; Bray-Curtis distance) suggested that both Bacteria and Fungi communities differed on the basis of sample type, as demonstrated by the separation of points on the ordination plane, roughly based on sample type groups (i.e. see color of points) as showed in Figs. 2a, b, c and 3a, $\mathrm{b}, \mathrm{c}$. When all the samples were included in the analysis, bacterial community on nest paper clearly differed compared to all other sample types (Fig. 2a), while the same condition was less evident for Fungi (Fig. 3a), where a partial overlap between nest paper and meconium (i.e. the not-living sample) was evident. Despite all cross-comparisons between samples type were significant, nest paper sample showed the most pronounced differences compared to all other samples (Figs. 2d and 3d, see color intensity of the first column in the heatmap of $R^{2}$, reported for all pairwise PERMANOVA comparisons). In addition, nest paper samples showed high within-sample similarity in community structure, as suggested by the complete overlap of samples coming from different nests in the ordination plane (Figs. 2a, b and 3a, b, see the position of the blue points in overlap to each other in the PCoA; Figs. 2e, 3e, see the high level of similarity showed by Sorensen index, as indicated by the yellow color, and the value distributions represented by ridgeline plot in the nest-nest cross-comparison). On the other hand, a clear separation on the basis of developmental stage can be observed when only living samples were considered (Figs. 2c and 3c), showing a sharp stage -related clusters. Overall, adult stages (i.e. workers and gynes) appeared separated from immature stages (i.e. larvae and newly emerged) as displayed by the first PCoA (Figs. 2c, 3c, explaining 21.4 and $20.2 \%$ of variance, respectively) and hierarchical clustering analysis (UPGMA method based on Bray-Curtis distance; Figs. 2f, 3f), 


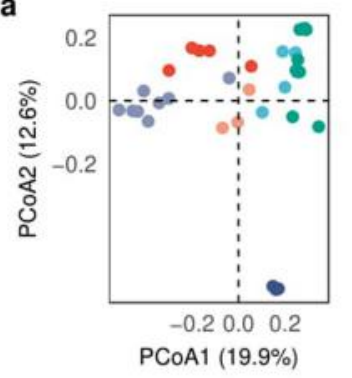

b

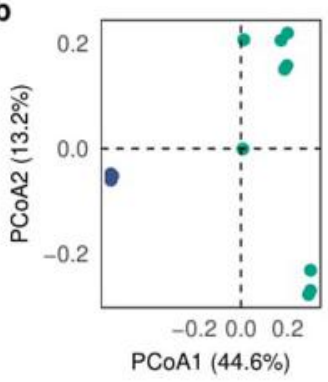

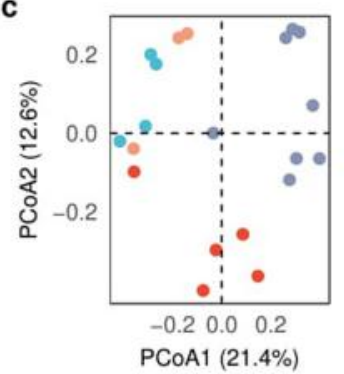

d

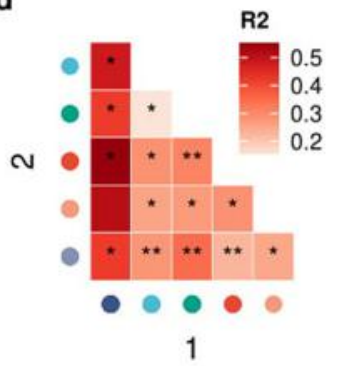

Sample type

gyne

larva

meconium

nest

newly_emerged

worker

e gyne

larva

meconium

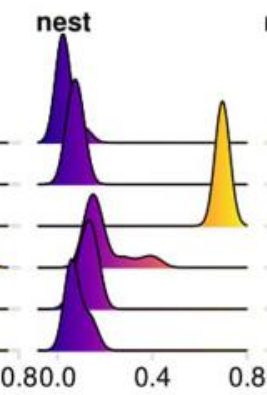

newly_emerged worker
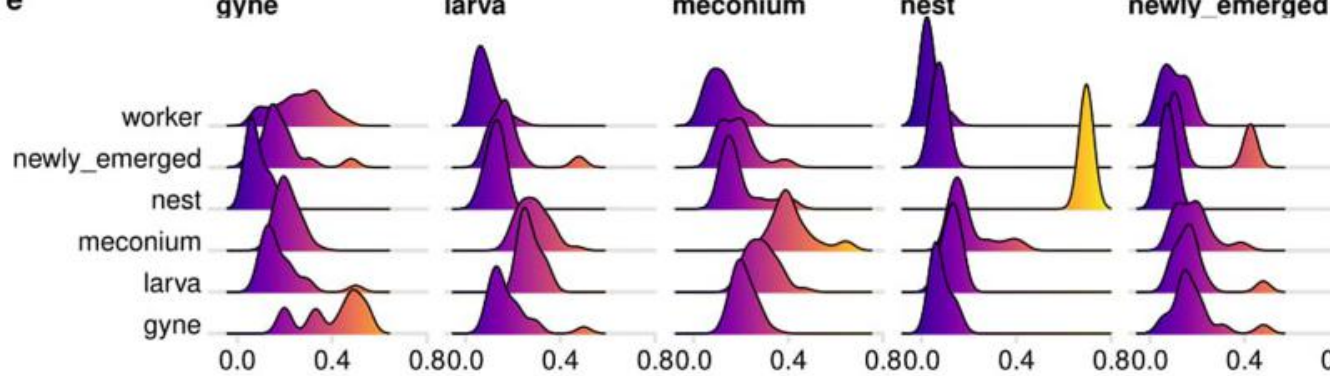

f

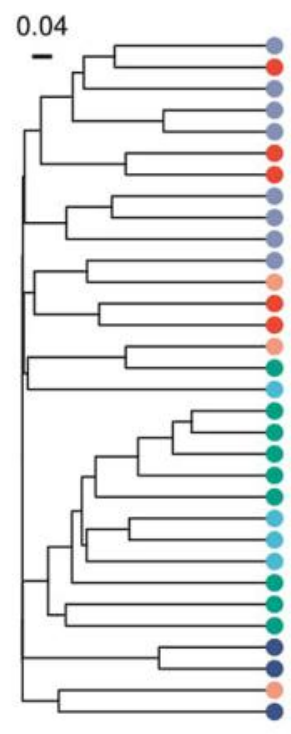

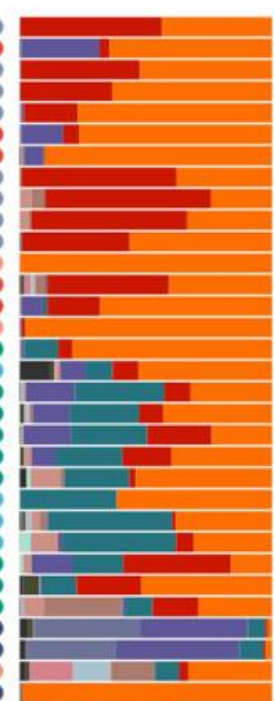

g
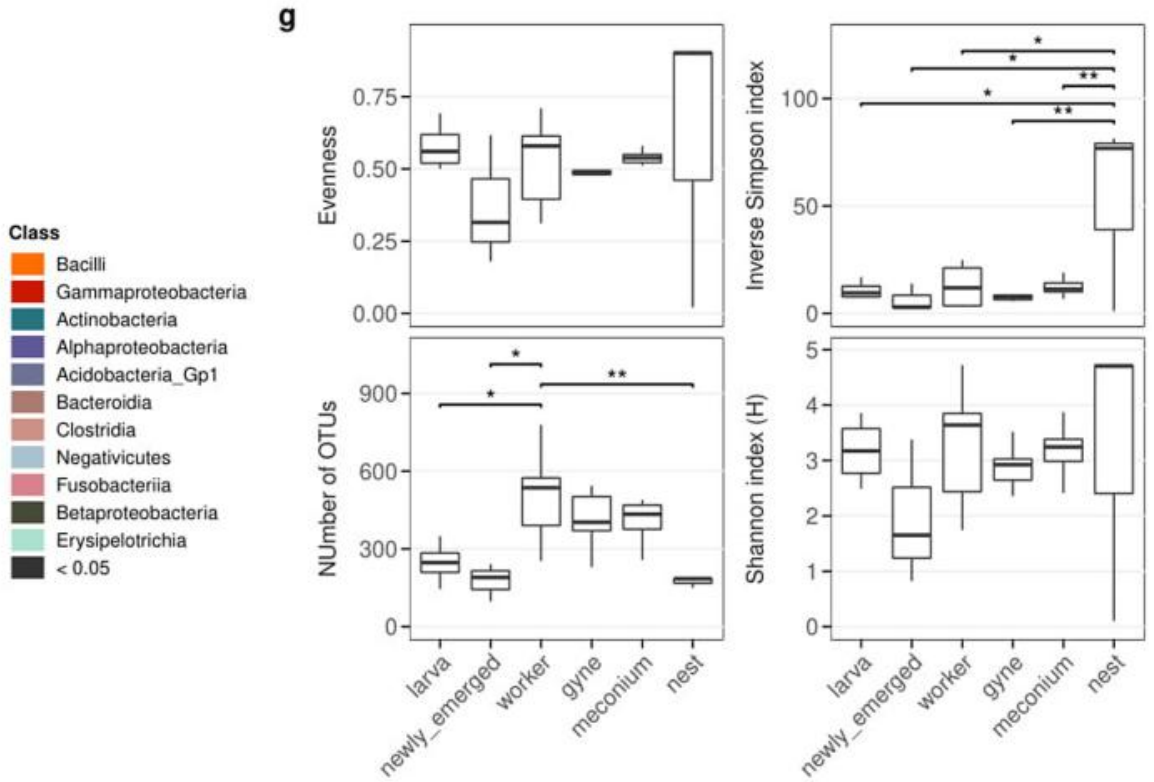

Sorensen index

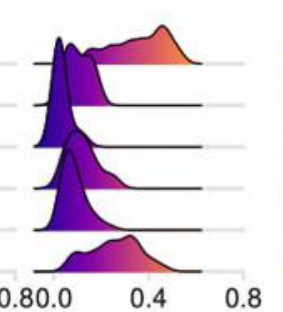

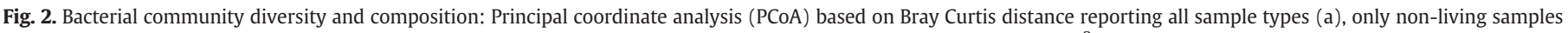

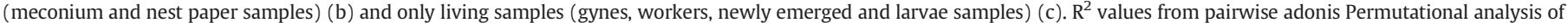

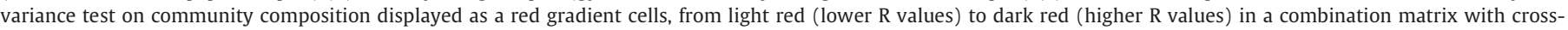

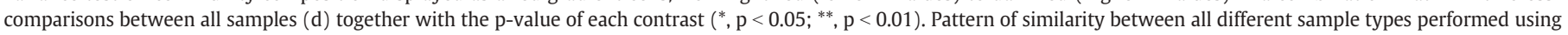

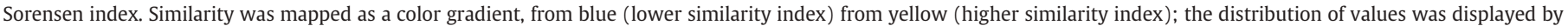

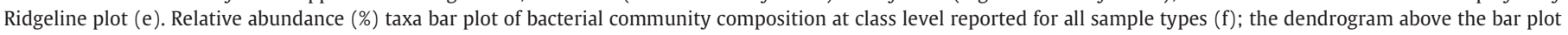

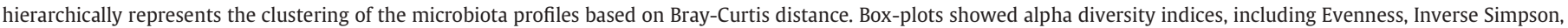
Number of OTUs and Shannon diversity $(\mathrm{g})(* \mathrm{p}<0.05, * * \mathrm{p}<0.01)$.

and this was especially evident for Bacteria, while Fungi seemed to hold a more stage-specific community signature.

Results from PCoA were in accordance with beta diversity analysis performed using Sorensen similarity index that showed profiles of similarity based on the distribution of values from Sorensen index plotted by ridgeline plot that showed a cross-comparison between all sample groups (Figs. 2e and 3e). Panel e of Figs. 2 and 3 shows the distribution of the Sorensen index for all sample types. The analysis showed a distribution similar to the one reported in the PCoA analysis (panels a, b, and c of Figs. 2 and 3). Nest paper samples were highly conserved (i.e. contrasts within samples of the same type reported a high similarity level whereas they displayed a high level of dissimilarity compared to other sample types). Gynes paired in similarity with newly emerged females and the same condition was visible for larvae and meconium. Gynes and newly emerged females showed more shared phylotypes compared to the other samples and the same condition was reported for larvae and meconium (Sorensen similarity index, Figs. 2 and 3 panel e).

Sample type affected alpha-diversity indices though the effect was more relevant regarding the mycobiota (Table 2) and no significant effect has emerged considering the association with the nest (origin). Alpha diversity indices in fungi were much more influenced by the sample type variable than Bacteria, indicating that yeasts constitute a better signature of gut microbiota comparing to the Bacteria. The worker and gyne stages resulted to be dominated by Saccharomycetes that 

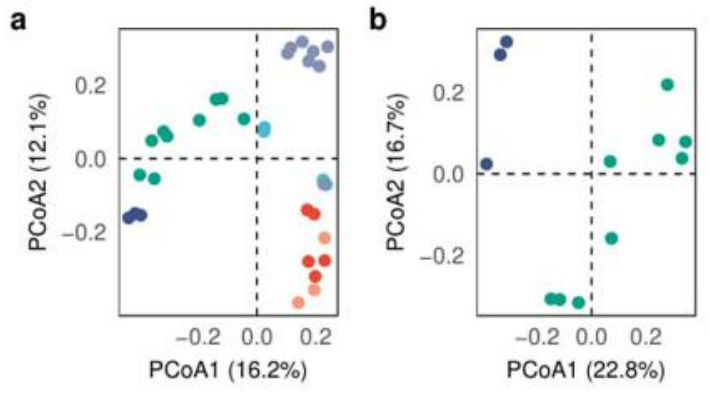

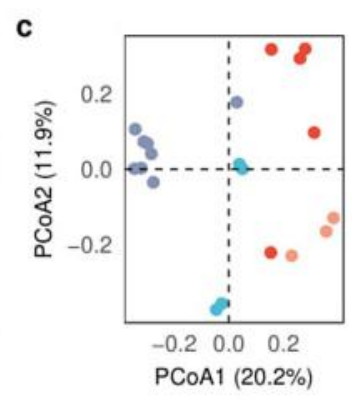

d

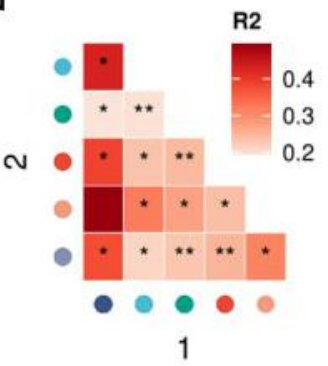

\section{Sample type}

gyne

- larva

- meconium

- nest

newly_emerged

worker<smiles>[124IH]</smiles>
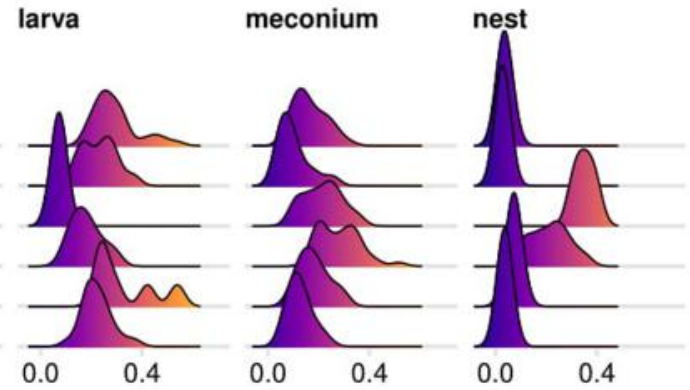

newly_emerged worker

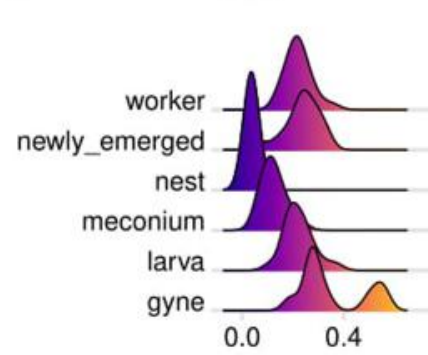

0.0

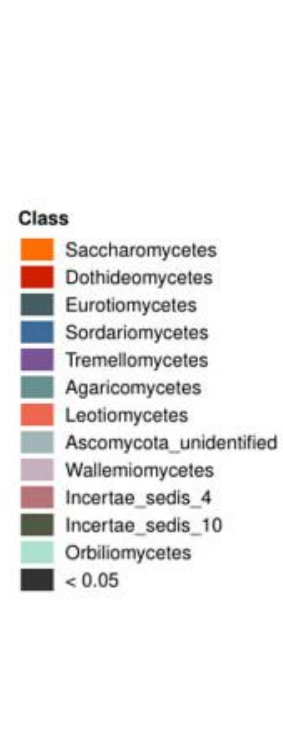

g
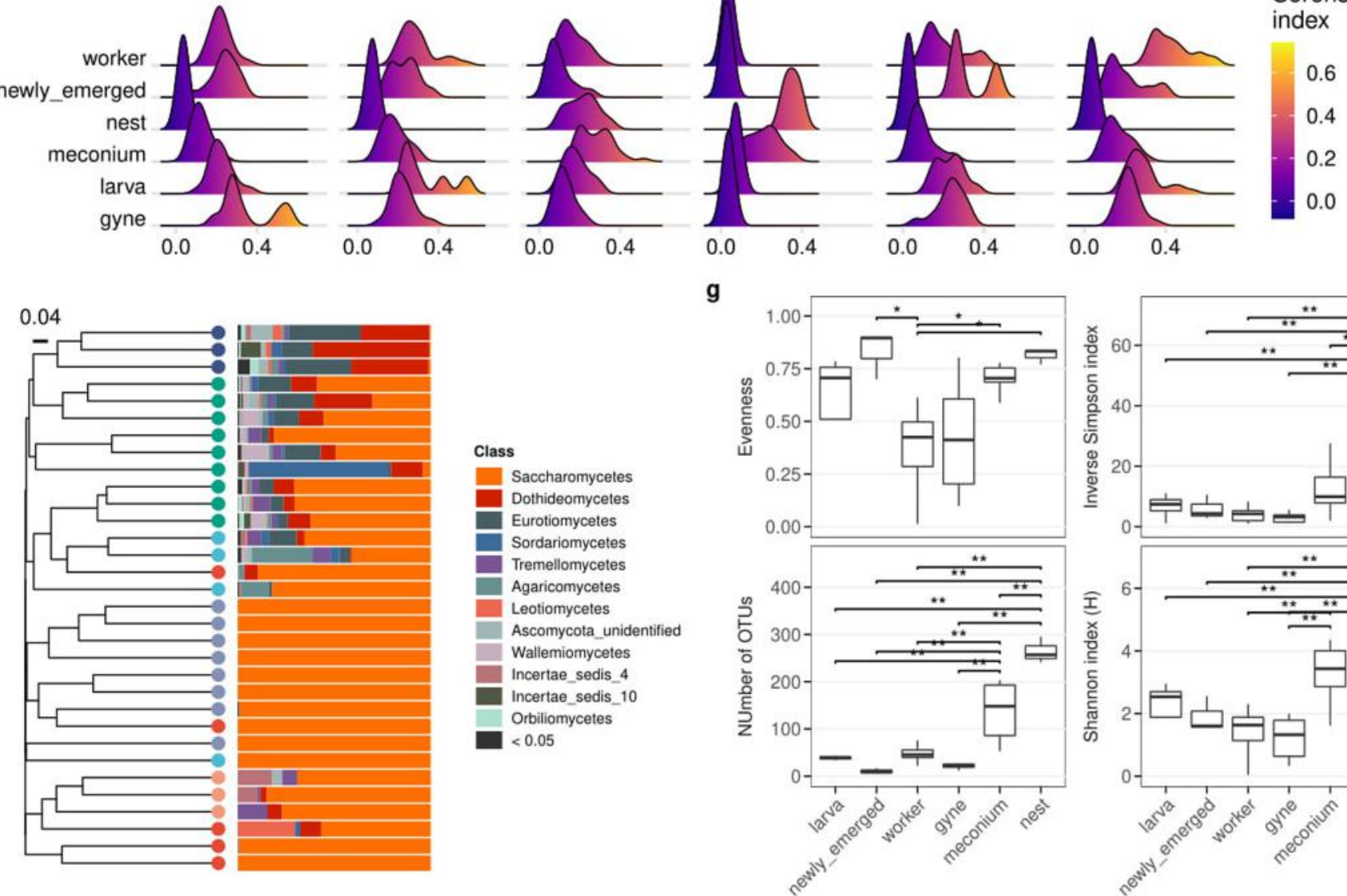

Sorensen
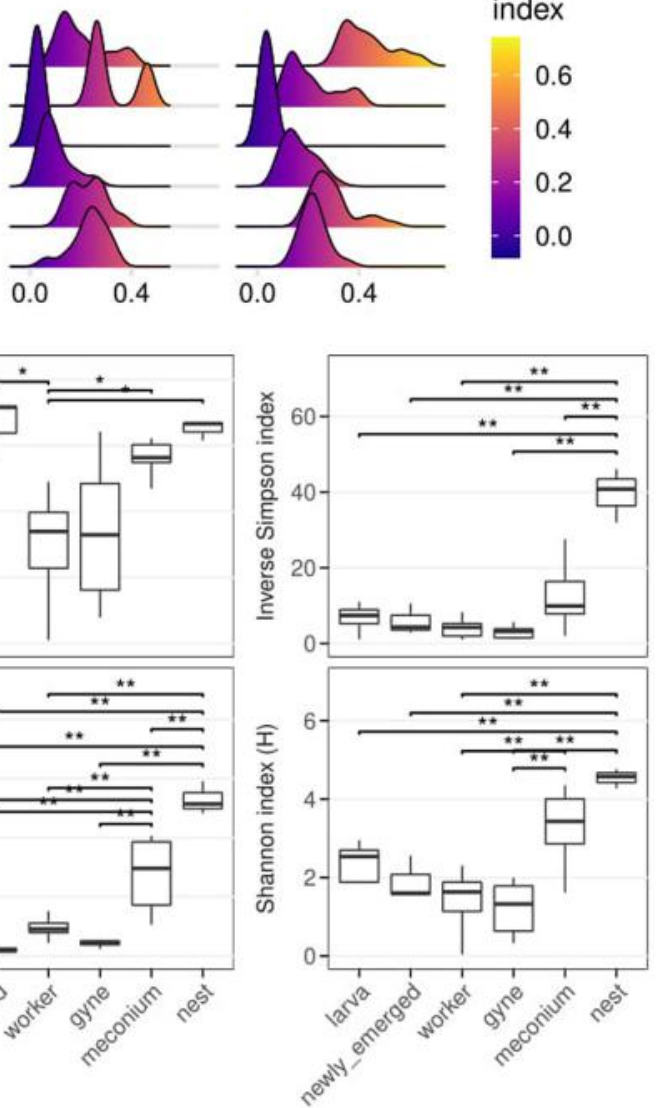

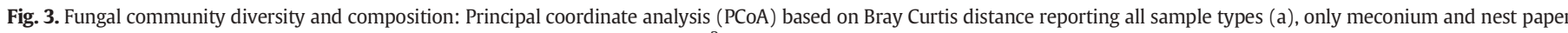

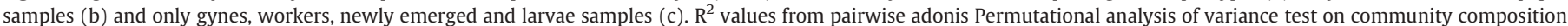

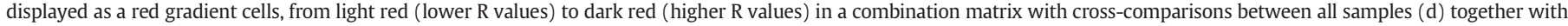

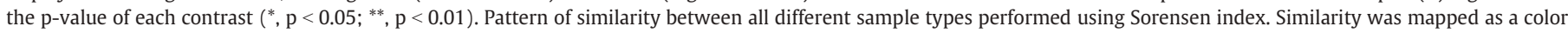

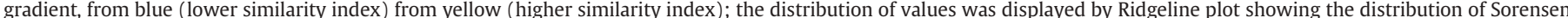

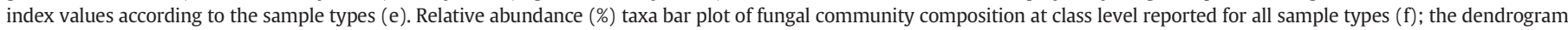

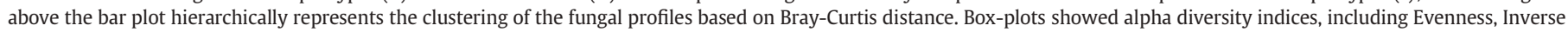
Simpson, Number of OTUs and Shannon diversity $(\mathrm{g})(* \mathrm{p}<0.05, * * \mathrm{p}<0.01)$.

Table 1

PERMANOVA performed on the sample type for both bacterial and fungal communities.

\begin{tabular}{rlrclll}
\hline & & Df & SumOfSqs & $\mathrm{R}^{2}$ & $\mathrm{~F}$ & $\operatorname{Pr}(>\mathrm{F})$ \\
\hline \multirow{2}{*}{$16 \mathrm{~S}$} & Sample type & 5 & 5.240 & 0.459 & 4.417 & $<0.001$ \\
& Residual & 26 & 6.169 & 0.541 & $/$ & $/$ \\
& Total & 31 & 11.409 & 1 & $/$ & $/$ \\
\multirow{4}{*}{ TTS } & Sample type & 5 & 4.632 & 0.401 & 3.486 & $<0.001$ \\
& Residual & 26 & 6.910 & 0.599 & $/$ & $/$ \\
& Total & 31 & 11.542 & 1 & $/$ & $/$ \\
\hline
\end{tabular}

hierarchically decreased in newly emerged, larvae, meconium, and finally appear almost absent in nest paper samples. On the other side, the most represented bacterial classes across all samples results to be the following class, Bacilli and Gammaproteobacteria mainly represented in workers, gynes and newly emerged.

Workers showed a significant higher richness rank compared to the meconium, newly emerged and nest paper samples but this does not result in a highest level of diversity compared to the other sample types (Fig. 2g). Richness and diversity of bacterial communities significant differed among sample types, with nest communities showing higher 
Table 2

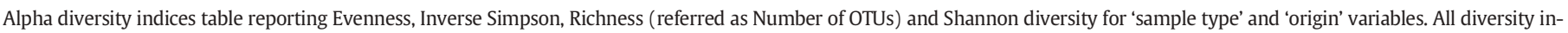
dices were reported for both $16 \mathrm{~S}$ and ITS datasets. ( ${ }^{*} \mathrm{p}<0.05,{ }^{* *} \mathrm{p}<0.01$ ).

\begin{tabular}{|c|c|c|c|c|c|c|c|c|c|}
\hline & Variable & Term & df & sumsq & meansq & Statistic & p.value & adj.p & Sign \\
\hline \multirow[t]{8}{*}{$16 S$} & Evenness & Sample type & 5 & 0.11 & 0.02 & 0.77 & 0.58 & 0.58 & \\
\hline & Evenness & Origin & 5 & 0.21 & 0.04 & 1.43 & 0.26 & 0.51 & \\
\hline & InvSimpson & Sample type & 5 & 4900.48 & 980.10 & 4.20 & 0.01 & 0.02 & $*$ \\
\hline & InvSimpson & Origin & 5 & 1140.05 & 228.01 & 0.98 & 0.46 & 0.46 & \\
\hline & Number of OTUs & Sample type & 5 & $428,144.98$ & $85,629.00$ & 5.71 & 0.00 & 0.00 & $* *$ \\
\hline & Number of OTUs & Origin & 5 & $61,735.93$ & $12,347.19$ & 0.82 & 0.55 & 0.55 & \\
\hline & Shannon diversity & Sample type & 5 & 4.41 & 0.88 & 0.84 & 0.53 & 0.53 & \\
\hline & Shannon diversity & Origin & 5 & 6.15 & 1.23 & 1.18 & 0.35 & 0.53 & \\
\hline \multirow[t]{8}{*}{ ITS } & Evenness & Sample type & 5 & 0.90 & 0.18 & 4.57 & 0.01 & 0.01 & $*$ \\
\hline & Evenness & Origin & 5 & 0.35 & 0.07 & 1.77 & 0.16 & 0.16 & \\
\hline & InvSimpson & Sample type & 5 & 3429.66 & 685.93 & 11.21 & $2.33 \mathrm{E}^{-05}$ & $4.65 \mathrm{E}^{-05}$ & $* *$ \\
\hline & InvSimpson & Origin & 5 & 270.51 & 54.10 & 0.88 & 0.51 & 0.51 & \\
\hline & Number of OTUs & Sample type & 5 & $180,574.35$ & $36,114.87$ & 37.79 & $8.31 \mathrm{E}^{-10}$ & $1.66 \mathrm{E}^{-09}$ & $* *$ \\
\hline & Number of OTUs & Origin & 5 & 9512.39 & 1902.48 & 1.99 & 0.12 & 0.12 & \\
\hline & Shannon diversity & Sample type & 5 & 36.27 & 7.25 & 12.20 & $1.25 \mathrm{E}^{-05}$ & $2.50 \mathrm{E}^{-05}$ & $* *$ \\
\hline & Shannon diversity & Origin & 5 & 5.37 & 1.07 & 1.81 & 0.16 & 0.16 & \\
\hline
\end{tabular}

differences compared to all other sample types, as displayed by Inverse Simpson index (Fig. 2g).

\subsection{Relative abundance and taxonomic distribution}

We detected differential relative abundant OTUs among all sample types using DESeq2 likelihood-ratio-test (LRT, alpha $<0.05$ ). Lactobacillus and Bifidobacterium showed the most differently abundant pattern mainly represented in gynes, larvae, and meconia (Fig. 4 G, L, M). Similarly, Lactococcus was one of the most represented genera, together with Lactobacillus and Bifidobacterium. Actinobacteria (Bifidobacterium, Alphaproteobacteria) and genera inside Bacilli phylum (Lactobacillus, Fructobacillus, Leuconostoc, Enterococcus, Staphylococcus, Streptococcus and Bacillus), with the exception of Lactococcus, represented the most abundant pattern associated with meconia. Furthermore, it showed a similar abundance distribution of Betaproteobacteria, Clostridia, and genera from Gammaproteobacteria (Frischella, Gilliamella and Diplorickettsia), thus representing the microbial communities displayed among all sample types (Fig. 4). Larvae and meconia showed the most similar pattern of abundance, even if the larval samples were less rich in Alphaproteobacteria, Betaproteobacteria, Gilliamella and Diplorickettsia (Fig. $4 \mathrm{~L}, \mathrm{M}$ ). Interestingly, newly emerged females and nest paper samples showed the poorest relative abundance distribution of the entire dataset (Fig. 4 N, NE). Gyne and worker samples harbored as main abundant phyla Bacilli and Gammaproteobacteria (Figs. 2f and 3f), however, both showed some quite distinct imbalance in characteristic differentially abundant taxonomic groups. Indeed the workers were less abundant in Lactobacillus, Alphaproteobacteria and Actinobacteria but richer in the genera from phylum Gammaproteobacteria, such as Buttiauxella (Fig. 4 G, W). Looking inside Gammaproteobacteria, overall mainly represented in workers, we found as the most abundant taxa, Klebsiella, Enterobacter and Escherichia/Shigella, also widely represented in gynes with the exception of Buttiauxella, one of the most abundant genus reported in worker samples (Fig. $4 \mathrm{G}, \mathrm{W}$ ). Concerning the genera inside Bacilli class, it is noteworthy the difference between adult stages (gynes and workers), with respect to the larvae and meconium samples. In fact, a different abundance distribution of taxa classified as genus Lactobacillus was evident among those groups (with most Lactobacillus taxa in larvae and meconial), as well as a substantial depletion of OTUs from the Lactococcus genus in meconium and larvae.

DESeq2 likelihood-ratio-test (LRT, alpha $<0.05$ ) performed on fungal communities showed Saccharomycetes as the main representative class of the entire ITS dataset. Metschnikowia and Hanseniaspora thailandica proved to be widespread across all sample types, even if they resulted to be less abundant in the nest paper samples (Fig. $5 \mathrm{~N}, \mathrm{E}$ ). The analyses also showed differentially abundant species within sample types,
Metschnikowia cibodasensis absent in gynes resulted mostly related to meconium and workers, while less present in the other sample types. Pichia fermentans was one of the main abundant genera in the workers, it decreased in meconium and gynes, while it was absent in newly emerged individuals and nest paper samples (Fig. 5 M,G,NE). Debaryomyces prosopidis was strongly represented in larvae, meconium and worker samples; similarly Saccharomycetales order mirrors this abundance pattern with the exception for gynes; where this taxa was strongly represented. Tremellomycetes were the only Basidiomycetes in the entire ITS dataset showing a high abundance level in meconium, larvae, nest and in only one gyne sample. As it happened for the microbiota, also in the mycobiota the meconium was still the most representative sample, in term of diversity, of the entire stage dataset.

\section{Discussion}

Here we provided the first comprehensive characterization of the bacterial and fungal communities of an introduced European population of the Asian hornet $V$. velutina nigrithorax. We carried out an extensive metataxonomic investigation, describing both the microbiota and mycobiota communities of different hornet life stages (larvae, newly emerged and adult females), including two different reproductive phenotypes (workers and gynes). Our analysis also included two types of not-living colonial samples, namely the meconial and the nest material (referred as nest paper).

Overall, we identified Bacilli, Gammaproteobacteria, Actinobacteria, Alphaproteobacteria as the most abundant classes of Bacteria, and Saccharomycetes, Dothideomycetes, Eurotiomycetes, Sordariomycetes and Tremellomycetes as the most abundant Fungi classes. Alpha diversity indices showed that the worker stage harbours a significantly higher number of Bacterial OTUs compared to all other sample types, however there are no differences displayed by Shannon and Evenness indices among all sample types, except for Inverse Simpson index that suggests a significantly higher diversity composition of the nest compared to the other sample types. Nevertheless, the alpha diversity indices suggest that the number and distribution of bacterial species among all sample types are rather uniform, with the exception of some significant differences as shown in Figs. $2 \mathrm{f}$ and $3 \mathrm{f}$. Similarly, the same indication is displayed in the alpha diversity indices analysis of Fungi community composition that follows the trend described above but includes meconium as well as nest sample. Overall, alpha diversity was affected by the sample type and not by the colony of origin.

A striking result of this study is that microbial communities showed significant sample-specific signature for both Bacteria and Fungi, showing differences in composition across life stages and among reproductive phenotypes. Indeed, beta diversity analyses showed a pattern of 


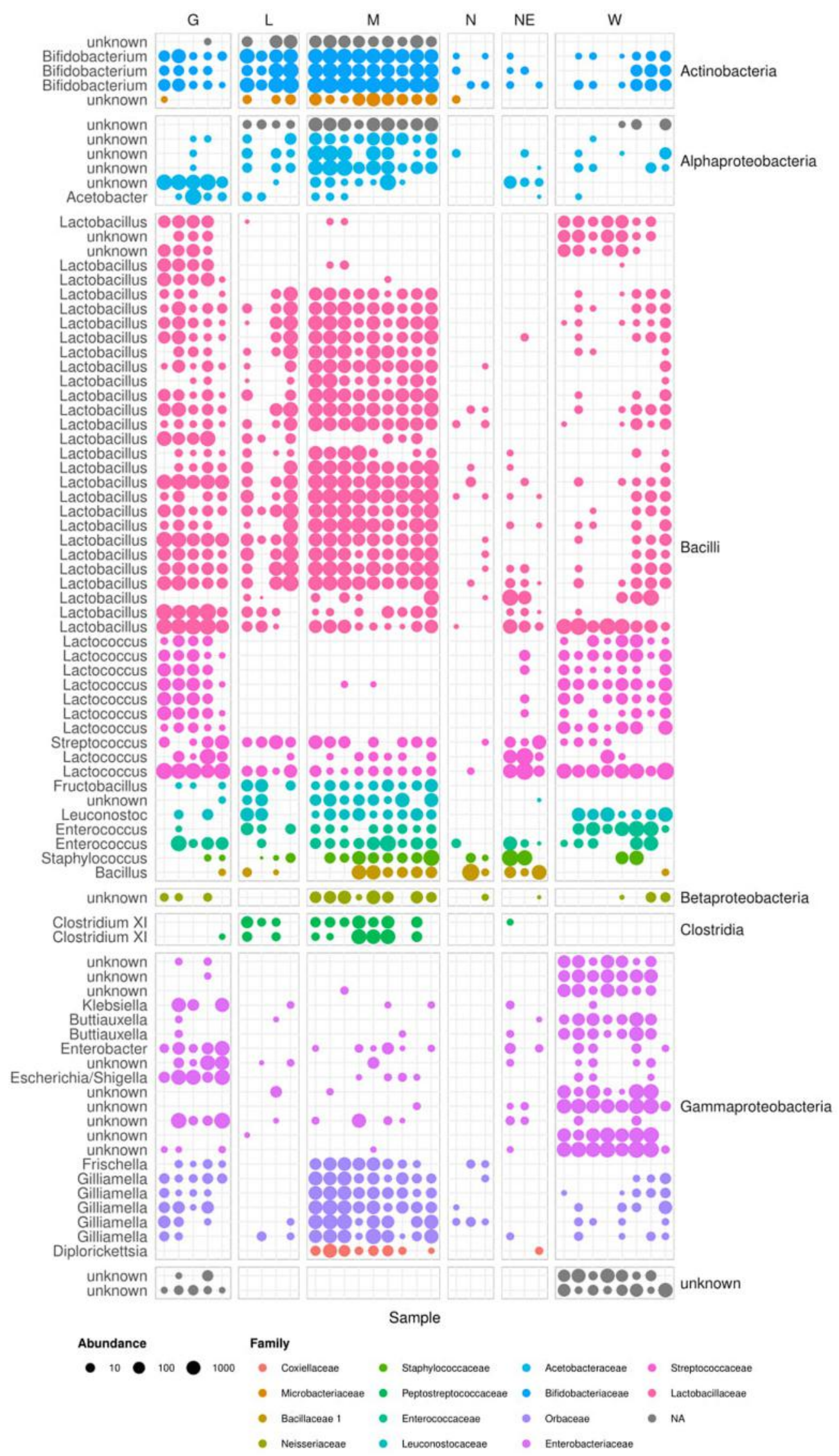

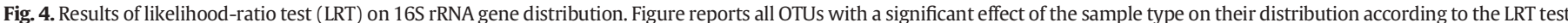

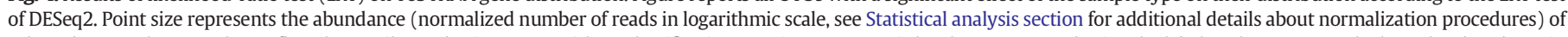

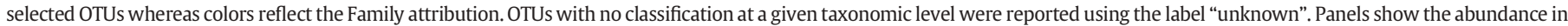
all sample types: G: gynes, L: larvae, M: meconia, N: nests, NE: Newly emerged individuals, W: workers. 


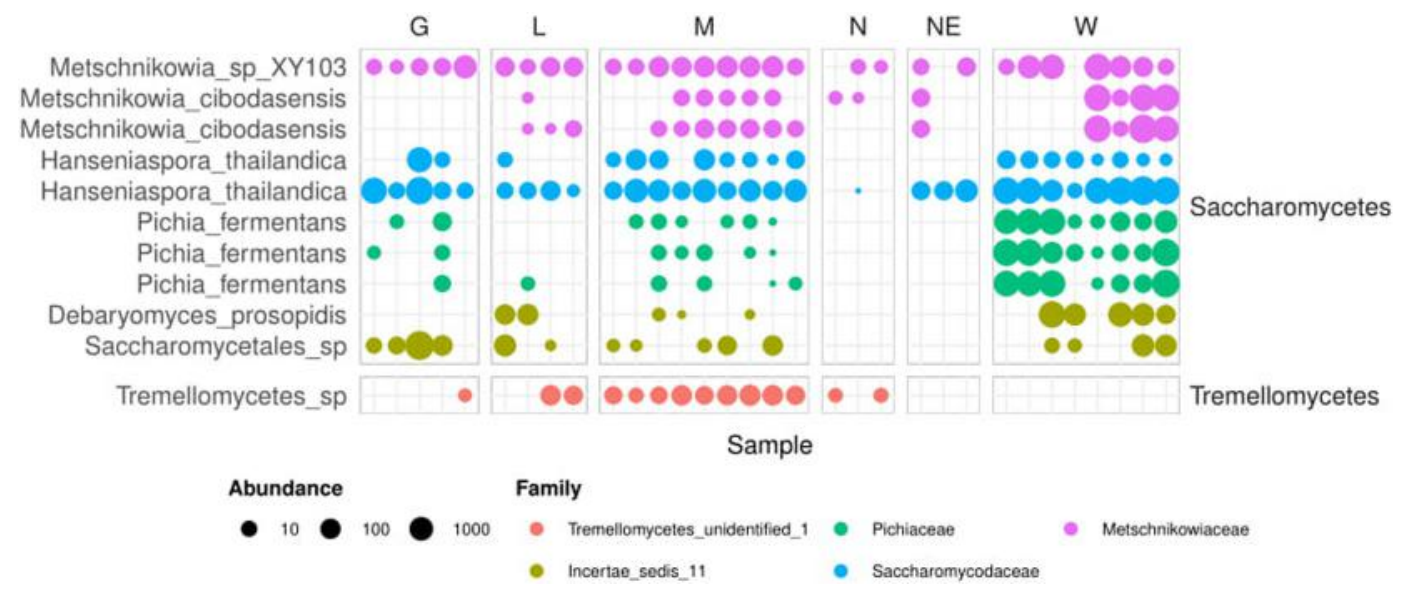

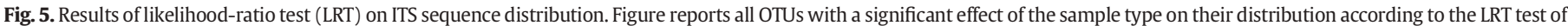

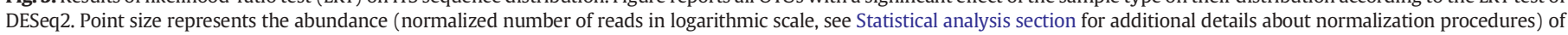

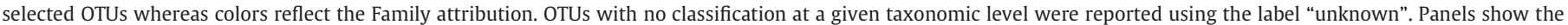
abundance in all sample types: G: gynes, L: larvae, M: meconia, N: nests, NE: Newly emerged individuals, W: workers.

sample-related clustering composition among all sample types in agreement with the specific life-style of each developmental stage, indicating the presence of sample-type related microbiota and mycobiota. Moreover, all sample's community composition grouped according to the hornet's phenotypic change during ontogeny, showing a displacement of the communities along a succession from the larval stage and the meconium up to the adult phase (workers and gynes), passing through the newly emerged females. We can therefore speculate that the ontogenetic development of hornets from the larva to the adult stages leads to a clear change in the intestinal microbial diversity and composition. The adult stages (gynes and workers) show an increase in gut bacterial richness (e.g. number of OTUs), suggesting that the hornet gut microbiota reaches the higher level of bacterial load in the adult stage rather than during the larval stage. The striking difference in diet of adults (sugar-based) and larvae (protein-based) is likely to play a role and needs to be investigated in the future as a possible driver of such variation.

The main bacterial classes found in living samples were Bacilli and Gammaproteobacteria. Actinobacteria are mostly present in the meconial samples, they decrease in larvae and adult stages. The presence of Actinobacteria in larval stages may play a role in the developmental process or nutrients digestion and defence against parasites. Cases of Actinobacteria symbionts have been found in pyrrhocorid bugs demonstrating their role in the sterilization of the egg surface (Salem et al., 2013). Different phylotypes of Actinobacteria have been shown to defend ants, beetles and wasps against detrimental microorganisms through the production of antibiotics (Kaltenpoth, 2009; Seipke et al., 2012; Visser et al., 2012) and they might also be involved in the nutrient processing and acquisition (Hanshew et al., 2014; Salem et al., 2014). Orbaceae, Lactobacillus and Acetobacteraceae were reported as the main enriched families in adult stages (gynes and workers). As previously found in Drosophila melanogaster, bacterial communities affected by fruit-sugar rich diet were also involved in the maintenance of the immune system and mating preferences (Broderick and Lemaitre, 2012). These communities are often associated with a fruit-based diet, which agrees with the hornet lifestyle. Indeed, adult hornets are essentially generalist sugarfeeding insects and the main carbohydrate sources are: tree sap, honeydew, flower nectar, ripe fruit, mushrooms and other sugary secretions (Matsuura and Yamane, 1990), while larvae are carnivorous and feed on arthropods such as bees, preyed by adult stages.

Interestingly, bacterial genera associated with bees, such as Frischella and Gilliamella, appeared heavily enriched in the meconium samples (probably due to the accumulation effect, as the meconium is made up of several layers of larvae faeces). This is probably because these microorganisms acquired with the diet (predation on bees) are "transient" in the gut of the larvae accumulating in the meconium stratifications. Their presence in adult stages (gynes and workers), quantitatively lower than in the meconium, might be explained by predation on bees.

Bifidobacterium and Lactobacillus were the main abundant genera displayed in our dataset. Already extensively described in Apis mellifera as the main bacterial communities responsible for a subset of functions, they are essential for the insect development and overall for the maintenance of the health of the entire colony (Forsgren et al., 2012). Here, Bifidobacterium and Lactobacillus were transversally distributed through the insect samples but mostly abundant in the adult stages, consolidating their presence as one of the leading bacterial communities in the gut of social Hymenoptera. However, the same result was not described in the gut microbiota of $V$. velutina sampled in Korea as reported in Kim et al., 2018. The Asian hornets sampled in Korea did not showed the communities described above, instead they were mainly characterized by the genera Flavobacterium (24\%), Aeromonas (17\%), Pseudomonas (13\%) and Pedobacter (12\%) (Kim et al., 2018). The only genera shared between our dataset and that of Kim et al. were Enterobacter and Clostridium, however, in the work by Kim et al., these phylotypes were minimally represented $(0.08 \%$ and $0.01 \%$, respectively), reinforcing the substantial dissimilarity of the microbiota shown by the Korean and European Vv populations.

The most striking discovery is that the fungal component of the microbiome is the major driver of change, clearly differentiating during the different life stages. Variation in the microbial diversity indices linked to the sample types were much more related to the Fungi than Bacteria, qualifying the Fungi as a better signature of the different life stages. Results of $V$. velutina mycobiota in workers and gynes stages were mainly characterized by Saccharomycetes, which are conversely poorly represented in paper nest and meconium. Instead, the paper nest and meconium are dominated by Dothidomycetes, Tremellomycetes and Eurotiomycetes. The nest material showed a completely different fungal community composition compared to the other sample types as displayed by diversity and composition analyses.

We can assert that the hornet gut mycobiota, as well as the microbiota, was not affected by the nest-associated microbial communities. This aspect is extremely interesting given that the nest represents the environment where the larvae grow and develop into adult stages and take part in the expansion, nursing and maintenance of the colony. The diet is potentially the best explanation for the finding that Saccharomycetes are most abundant in adult stages, as adult hornets feed on sugary sources, such as fruits. This reinforces the importance of the Hymenoptera in the dispersion and overall in the ecology of these sugar-related yeast (Stefanini, 2018). 
The main relatively abundant taxa representative of the ITS dataset were Metschnikowia cibodasensis, Hanseniaspora thailandica, Pichia fermentans, Debaryomyces prosopidis, Metschnikowia, Saccharomycetales and Tremellomycetes.

Pichia fermentans is an Ascomycete known for its fermentative traits and its presence in many fermentative substrates but recently was also found in association with insects, such as D. melanogaster and social wasps (Fogleman et al., 2014; Stefanini et al., 2012). This makes $P$. fermentans a yeast capable of living and adapting to the gut of different insect species. P. fermentans, as well as yeast from genera Metschnikowia, and Hanseniaspora, are often isolated from fruit trees (Vadkertiová et al., 2012), in accordance with the diet of the adult stages, which feed on sugary sources such as fruits, thus sharing the adherent microorganisms and providing fermentable substrate to those already present. These results support the hypothesis that the hornet gut mycobiota is affected by the life stage-related diet. Indeed, after the elimination of the peritrophic sac at the end of immature life, the intestine shows a low number of communities (as we found in the newly emerged stage), and then it can be easily colonized. This might explain the process of colonization of the intestinal tract by Saccharomycetes and their strong predominance in the adult stages.

Tremellomycetes, found in the nest paper samples and in the meconium, is known as a class that includes plant pathogen strains (Weiss et al., 2014). The presence of Tremellomycetes in the nest paper is in accordance with the nest building matrix usually made by environmental wood debris and this offers interesting insights to consider the analysis of the nest microbial composition as a biomarker assay in the study of plant pathogens.

Finally, the discovery that the species' core microbiota in adults and larvae differ almost completely with the nest's microbiota, suggests that the social wasps have a proprietary microbiome. In addition to providing shelter and protection for the brood, the nest also represents the locus of Vespidae social life (Starr, 2019). At the same time, the nest is a product of wasp activity (the paper of the nest is made by vegetal fibres chewed by wasps) and is filled with hornet products, the principal of which is the meconium, for instance, the residues of larval faeces. Thus, our findings that nest's microbiome does not reflect the microbiota harbored by hornet guts indicates that the environment in which hornets live most of their life is not a source of the microbes that make the hornet's microbiome. The evolution of microbial communities during ontogenetic development was previously described in other social insects, such as Apis mellifera (Martinson et al., 2012). Our results confirm the findings that variation in the physiochemical conditions of the gut in invertebrates at different life stages are a driver of development of microbial communities (Lemke et al., 2003). Our results provide the "first metagenomic resource" of the microbiome of $V$. velutina in Europe, and also suggest the importance of considering life stages and reproductive phenotypes as well as nest influence in order to obtain a comprehensive picture of insect microbial communities. The detailed information we provide is instrumental to enable future comparisons with native or non-invasive hornet species, paving the way to understand the microbial features that might influence species success and impact on ecosystems.

This metagenomic resource is also a promising tool for the development of pest management strategies. Indeed, the understanding of the healthy intestinal microbiome, in terms of diversity and functionality, is the first step for the management approach which uses the manipulation and the exploitation of insect microbiota in order to reduce pest insectrelated problems. Understanding the microbial composition and diversity of the pest species is crucial for two reasons (Douglas, 2007; Arora and Douglas, 2017). First, it allows us to understand how to disrupt the microbial community of the pest species by targeting key components, thus reducing insect pest fitness. A similar approach has been recently proposed to control termites by using genetically engineered yeast as a 'TrojanHorse' that kills the cellulose-digesting protozoa in the termite gut (Sethi et al., 2014). Second, a knowledge of the pest microbial community allows us to understand which microbial components have major impacts on the traits contributing to its pest status (e.g. capacity to vector diseases, natural enemy resistance and host range). Interfering with these components has the potential to reduce the negative impacts of the pest (as it has been shown with the case of the mosquito Aedes aegypti, where the experimental transfer of Wolbachia suppresses the host vector competence, reviewed in Arora and Douglas, 2017). To our knowledge this approach has never been developed for hornets, and our metagenomic characterization of the gut microbial community in Vv may represent a first step toward this goal.

Notwithstanding the somehow limited number of samples and colonies investigated due to the difficulty of working with an alien species under local eradication programs, the results of this study provides a springboard for future assessment of the functional role of gut microbial organisms in hornets. In particular, our microbial characterization indicates specific fungal and bacterial groups associated with specific life stages, thus calling for future assessment of their role in the hornet survival and life-history traits. Moreover, our results represent a first step in the long road to link gut microbiota to the role played by hornets in ecosystems, as well as also enabling a first step toward possible management strategies of this invasive pest. Understanding the microbial composition which is essential to Vv survival has the potential to indicate the target of future microbial control of this pest species.

\section{CRediT authorship contribution statement}

Alessandro Cini: Methodology, Investigation, Writing - original draft, Writing - review \& editing. Niccolò Meriggi: Methodology, Formal analysis, Writing - original draft, Writing - review \& editing. Giovanni Bacci: Formal analysis, Writing - review \& editing. Federico Cappa: Methodology, Investigation, Writing - review \& editing. Francesco Vitali: Methodology, Formal analysis, Writing - review \& editing. Duccio Cavalieri: Methodology, Writing - review \& editing. Rita Cervo: Methodology, Writing - review \& editing.

\section{Declaration of competing interest}

The authors declare no conflict of interest/Not applicable.

\section{Acknowledgements}

We wish to thank Fabrizio Zagni and Nuccio Lanteri for their help in collecting combs and hornet samples, Iacopo Petrocelli and Irene Pepiciello for help with dissections and sample preparation and Lewis Revely for a critical reading of an earlier version of the manuscript.

\section{Funding}

Funds are provided to RC the project ALIEM (Action pour Limiter les risques de diffusion des espèces Introduites Envahissantes en Méditerranée), funded by the 'Programma Interreg Italia-Francia Marittimo 2014-2020. -La cooperazione al cuore del Mediterraneo', axe II (grant number J12I16000080007). FC received a grant from the same project.

\section{Availability of data and material}

Microbial community composition data is available as supplementary material (for bacteria, files: OTUStable16s.csv, IDTable16s.csv, TaxaTable16s.csv; for fungi, files: OTUStableITS.csv, IDTableITS.csv, TaxaTableITS.csv). Sample sequences have been archived on the European Nucleotide Archive (ENA), accession number: PRJEB36629.

\section{Appendix A. Supplementary data}

Supplementary data to this article can be found online at https://doi. org/10.1016/j.scitotenv.2020.140873. 


\section{References}

Albanese, D., Fontana, P., De Filippo, C., Cavalieri, D., Donati, C., 2015. MICCA: a complete and accurate software for taxonomic profiling of metagenomic data. Sci. Rep. 5, 9743 https://doi.org/10.1038/srep09743.

Anderson, K.E., Ricigliano, V.A., 2017. Honey bee gut dysbiosis: a novel context of disease ecology. Curr. Opin. Insect Sci. 22, 125-132. https://doi.org/10.1016/j. cois.2017.05.020.

Andrews, S., 2010. FastQC: a quality control tool for high throughput sequence data. Babraham Bioinformatics (version 0.11.3) Available at. http://www.bioinformatics. babraham.ac.uk/projects/fastqc. (Accessed 25 March 2015).

Arora, A.K., Douglas, A.E., 2017. Hype or opportunity? Using microbial symbionts in nove strategies for insect pest control. J. Insect Physiol. 103, 10-17. https://doi.org/ 10.1016/j.jinsphys.2017.09.011

Berlanga, M., Paster, B.J., Grandcolas, P., Guerrero, R., 2011. Comparison of the gut microbiota from soldier and worker castes of the termite Reticulitermes grassei. Int. Microbiol. 14, 83-93. https://doi.org/10.2436/20.1501.01.138.

Broderick, N.A., Lemaitre, B., 2012. Gut-associated microbes of Drosophila melanogaster Gut Microbes 3 (4), 307-321. https://doi.org/10.4161/gmic.19896.

Brune, A., Dietrich, C., 2015. The gut microbiota of termites: digesting the diversity in the light of ecology and evolution. Annu. Rev. Microbiol. 69, 145-166. https://doi.org/ 10.1146/annurev-micro-092412-155715.

Cappa, F., Cini, A., Pepiciello, I., et al., 2019. Female body size, weight and fat storage rather than nestmateship determine male attraction in the invasive yellow-legged hornet Vespa velutina nigrithorax. Ethol. Ecol. Evol. 31 (1), 73-85. https://doi.org/10.1080/ 03949370.2018.1501437.

Cardoso, P., Leather, S.R., 2019. Predicting a global insect apocalypse. Insect Conserv. Divers. 12 (4), 263-267. https://doi.org/10.1111/icad.12367.

Chapman, R.E., Bourke, A.F.G., 2001. The influence of sociality on the conservation biology of social insects. Ecol. Lett. 4 (6), 650-662. https://doi.org/10.1046/j.14610248.2001.00253.x.

Chugo, S., Lizaso, M.T., Alvarez, M.J., et al., 2015. Vespa velutina nigritorax: a new causative agent in anaphylaxis. J Investig Allergol Clin Immunol 25, 231-232.

Cini, A., Cappa, F., Petrocelli, I. et al., 2018. Competition between the native and the introduced hornets Vespa crabro and Vespa velutina: a comparison of potentially relevant life-history traits. Ecol. Entomol. 43 (3), 351-362. https://doi.org/10.1111/een.12507.

Crotti, E., Balloi, A., Hamdi, C., et al., 2012. Microbial symbionts: a resource for the management of insect-related problems. Microb. Biotechnol. 5 (3), 307-317. https://doi. org/10.1111/j.1751-7915.2011.00312.x.

Dapporto, L., Stefanini, I., Rivero, D., et al., 2016. Social wasp intestines host the local phenotypic variability of Saccharomyces cerevisiae strains. Yeast 33 (7), 277-287. https:// doi.org/10.1002/yea.3173.

Del Toro, I., Ribbons, R.R., Pelini, S.L., 2012. The little things that run the world revisited: review of ant-mediated ecosystem services and disservices (Hymenoptera: Formicidae). Myrmecological News 17, 133-146.

Diouf, M., Roy, V., Mora, P., et al., 2015. Profiling the succession of bacterial communities throughout the life stages of a higher termite Nasutitermes arborum (Termitidae, Nasutitermitinae) using 16S rRNA gene pyrosequencing. PLoS One 10 (10), e0140014. https://doi.org/10.1371/journal.pone.0140014.

Douglas, A.E., 2007. Symbiotic microorganisms: untapped resources for insect pest control. Trends Biotechnol. 25 (8), 338-342. https://doi.org/10.1016/j. tibtech.2007.06.003.

Edgar, R.C., 2016. UNOISE2: improved error-correction for Illumina 16S and ITS amplicon sequencing. bioRxiv https://doi.org/10.1101/081257.

Engel, P., Moran, N.A., 2013. The gut microbiota of insects - diversity in structure and function. FEMS Microbiol. Rev. 37 (5), 699-735. https://doi.org/10.1111/15746976.1202-.

Fogleman, J.C., Starmer, W.T., Heed, W.B., Url, S., 2014. Comparisons of Yeast Florae From Natural Substrates and Larval Guts of Southwestern Drosophila. vol. 52, pp. 187-191. https://doi.org/10.1007/BF00363835.

Forsgren, E., Fries, I., Paxton, R.J., Flaberg, E., 2012. Symbionts as Major Modulators of Insect Health : Lactic Acid Bacteria and Honeybees. vol. 7, p. e33188. https://doi.org/ 10.1371/journal.pone.0033188 (3).

Granato, A., Negrisolo, E., Bonomi, J., et al., 2019. Recent confirmation of a single haplotype in the Italian population of Vespa velutina. Biol. Invasions 21 (9), 2811-2817. https:// doi.org/10.1007/s10530-019-02051-4.

Hanshew, A.S., McDonald, B.R., Díaz Díaz, C., et al., 2014. Characterization of Actinobacteria associated with three ant-plant mutualisms. Microb. Ecol. 69 (1), 192-203. https://doi.org/10.1007/s00248-014-0469-3.

Haxaire, J., Villemant, C., 2010. Impact sur l'entomofaune des pièges à Frelon asiatique. Insectes 159, 1-6.

Hughes, D.P., Pierce, N.E., Boomsma, J.J., 2008. Social insect symbionts: evolution in homeostatic fortresses. Trends Ecol. Evol. 23 (12), 672-677. https://doi.org/10.1016/j. tree.2008.07.011.

Joshi, N.A., Fass, J.N., 2011. Sickle: A Sliding-Window, Adaptive, Quality-Based Trimming Tool for FastQ Files (Version 1.33) [Software].

Kaltenpoth, M., 2009. Actinobacteria as mutualists: general healthcare for insects? Trends Microbiol. 17 (12), 529-535. https://doi.org/10.1016/j.tim.2009.09.006.

Kapheim, K.M., Rao, V.D., Yeoman, C.J., et al., 2015. Caste-specific differences in hindgut microbial communities of honey bees (Apis mellifera). PLoS One 10 (4), e0123911. https://doi.org/10.1371/journal.pone.0123911.

Kim, E., Seo, J., Yang, S.H., et al., 2018. Intestine bacterial microbiota of Asian hornet (Vespa velutina nigrithorax) and honey bee. Korean J. Environ. Agric. 37 (2), 135-140. https:// doi.org/10.5338/kjea.2018.37.2.18.
Koch, H., Schmid-Hempel, P., 2011. Socially transmitted gut microbiota protect bumble bees against an intestinal parasite. Proc. Natl. Acad. Sci. 108, 19288-19292. https:// doi.org/10.1073/pnas.1110474108.

Leather, S.R., 2018. "Ecological Armageddon" - more evidence for the drastic decline in insect numbers. Ann. Appl. Biol. 172, 1-3. https://doi.org/10.1111/aab.12410.

Lemke, T., Stingl, U., Egert, M., et al., 2003. Physicochemical conditions and microbial activities in the highly alkaline gut of the humus-feeding larva of Pachnoda ephippiata (Coleoptera: Scarabaeidae). Appl. Environ. Microbiol. 69, 6650-6658. https://doi. org/10.1128/AEM.69.11.6650-6658.2003.

Liu, Z., Chen, S., Zhou, Y., et al., 2015. Deciphering the venomic transcriptome of killerwasp Vespa velutina. Sci. Rep. 5 (9454), 9454. https://doi.org/10.1038/srep09454.

Love, M.I., Huber, W., Anders, S., 2014. Moderated estimation of fold change and dispersionfor RNA-seq data with DESeq2. Genome Biol. 15 (12), 550 (doi: 13059014-0550-8).

Martin, M., 2011. Cutadapt removes adapter sequences from high-throughput sequencing reads. EMBnet. J. 17 (1), 10-12.

Martinson, V.G., Moy, J., Moran, N.A., 2012. Establishment of characteristic gut Bacteria during development of the honeybee worker. Appl. Environ. Microbiol. 78, 2830-2840. https://doi.org/10.1128/aem.07810-11.

Matsuura, M., Yamane, S., 1990. Biology of the Vespine Wasps. Springer Verlag.

Meriggi, N., Di Paola, M., Vitali, F., et al., 2019. Saccharomyces cerevisiae induces immune enhancing and shapes gut microbiota in social wasps. Front. Microbiol. 10, 2320. https://doi.org/10.3389/fmicb.2019.02320.

Monceau, K., Thiéry, D., 2017. Vespa velutina nest distribution at a local scale: an 8-year survey of the invasive honeybee predator. Insect Sci. 24 (4), 663-674. https://doi. org/10.1111/1744-7917.12331.

Monceau, K., Bonnard, O., Thiéry, D., 2014. Vespa velutina: a new invasive predator of honeybees in Europe. J. Pest. Sci. 87, 1-16. https://doi.org/10.1007/s10340-013-0537-3.

Mutinelli, F., 2011. The spread of pathogens through trade in honey bees and their products (including queen bees and semen): overview and recent developments. Rev. Sci. Tech. 30, 257-271.

Nilsson, R.H., Larsson, K.-H., Taylor, A.F.S., Bengtsson-Palme, J., Jeppesen, T.S., Schigel, D. Kennedy, P., Picard, K., Glöckner, F.O., Tedersoo, L., Saar, I., Kõljalg, U., Abarenkov, K., 2018. The UNITE database for molecular identification of fungi: handling dark taxa and parallel taxonomic classifications. Nucleic Acids Res. 8 (47), D259-D264. https://doi.org/10.1093/nar/gky1022.

Oksanen, J., Blanchet, F.G., Friendly, M., Kindt, R., et al., 2019. vegan: Community Ecology Package. R package version 2.5-6. https://CRAN.R-project.org/package=vegan.

Otani, S., Zhukova, M., Koné, N.A., et al., 2019. Gut microbial compositions mirror castespecific diets in a major lineage of social insects. Environ. Microbiol. Rep. 11 (2), 196-205. https://doi.org/10.1111/1758-2229.12728.

Outhwaite, C.L., Gregory, R.D., Chandler, R.E., Collen, B., Isaac, N.J., 2020. Complex longterm biodiversity change among invertebrates, bryophytes and lichens. Nat. Ecol. Evol. 4, 384-392. https://doi.org/10.1038/s41559-020-1111-z.

Pielou, E.C., 1966. The measurement of diversity in different types of biological collections. Journal of theoretical biology 13, 131-144.

Pinto-Tomás, A.A., Anderson, M.A., Suen, G., et al., 2009. Symbiotic nitrogen fixation in the fungus gardens of leaf-cutter ants. Science 326 (5956), 1120-1123. https://doi.org/ 10.1126/science.1173036.

R Core Team, 2017. R: A Language and Environment for Statistical Computing. R Foundation for Statistical Computing, Vienna, Austria URL. https://www.R-project.org/.

Radek, R., 1999. Flagellates, bacteria, and fungi associated with termites: diversity and function in nutrition-a review. Ecotropica 5, 183-196.

Regulation, 2016. Commission Implementing Regulation (EU) 2016/1141 of 13 July 2016 adopting a list of invasive alien species of Union concern pursuant to Regulation (EU) No 1143/2014 of the European Parliament and of the Council. Off. J. Eur. Union, L 189 (4), 4-8

Requier, F., Rome, Q., Chiron, G., et al., 2019. Predation of the invasive Asian hornet affects foraging activity and survival probability of honey bees in Western Europe. J. Pest. Sci. 92 (2), 567-578. https://doi.org/10.1007/s10340-018-1063-0.

Robinet, C., Darrouzet, E., Suppo, C., 2018. Spread modelling: a suitable tool to explore the role of human-mediated dispersal in the range expansion of the yellow-legged hornet in Europe. Int. J. Pest Manag. 65 (3), 258-267. https://doi.org/10.1080/ 09670874.2018.1484529.

Rosengaus, R.B., Zecher, C.N., Schultheis, K.F., et al., 2011. Disruption of the termite gut microbiota and its prolonged consequences for fitness. Appl. Environ. Microbiol. 77 (13), 4303-4312. https://doi.org/10.1128/AEM.01886-10.

Salem, H., Kreutzer, E., Sudakaran, S., Kaltenpoth, M., 2013. Actinobacteria as essential symbionts in firebugs and cotton stainers (Hemiptera, Pyrrhocoridae). Environ. Microbiol. 15, 1956-1968. https://doi.org/10.1111/1462-2920.12001.

Salem, H., Bauer, E., Strauss, A.S., et al., 2014. Vitamin supplementation by gut symbionts ensures metabolic homeostasis in an insect host. Proc. R. Soc. B Biol. Sci. 7;281 (1796), 20141838. https://doi.org/10.1098/rspb.2014.1838.

Seipke, R.F., Barke, J., Ruiz-Gonzalez, M.X., et al., 2012. Fungus-growing Allomerus ants are associated with antibiotic-producing actinobacteria. Antonie van Leeuwenhoek, Int J Gen. Mol. Microbiol. 101 (2), 443-447. https://doi.org/10.1007/s10482-011-9621-y.

Sethi, A., Delatte, J., Foil, L., Husseneder, C., 2014. Protozoacidal Trojan-Horse: use of a ligand-lytic peptide for selective destruction of symbiotic protozoa within termite guts. PLoS One 9 (9), e106199. https://doi.org/10.1371/journal.pone.0106199.

Shannon, C.E., 1948. A mathematical theory of communication. Bell Syst. Tech. J. 27.

Simpson, E.H., 1949. Measurement of diversity. Nature 163 (4148), 688.

Spradbery, J.P., 1973. Wasps. An Account of the Biology and Natural History of Social and Solitary Wasps, With Particular Reference to Those of the British Isles.

Starr, C.K., 2019. 15. The nest as the locus of social life. In: Ross, K.G., Matthews, R.W. (Eds.), The Social Biology of Wasps. The Social Biology of Wasps. Cornell Univ. Press, Ithaca, pp. 520-539. 
Stefanini, I., 2018. Yeast-insect associations: it takes guts. Yeast, 315-330 https://doi.org/ 10.1002/yea.3309.

Stefanini, I., Dapporto, L., Legras, J.-L., et al., 2012. Role of social wasps in Saccharomyces cerevisiae ecology and evolution. Proc. Natl. Acad. Sci. 109, 13398-13403. https:// doi.org/10.1073/pnas.1208362109.

Suen, G., Scott, J.J., Aylward, F.O., et al., 2010. An insect herbivore microbiome with high plant biomass-degrading capacity. PLoS Genet. 6, e1001129. https://doi.org/ 10.1371/journal.pgen.1001129.

Takahashi, S., Tomita, J., Nishioka, K., Hisada, T., Nishijima, M., 2014. Development of a prokaryotic universal primer for simultaneous analysis of Bacteria and Archaea using next-generation sequencing. PLoS One 9 (8), e105592. https://doi.org/ 10.1371/journal.pone.0105592.

Vadkertiová, R., Molnárová, J., Vránová, D., Sláviková, E., 2012. Yeasts and yeast-like organisms associated with fruits and blossoms of different fruit trees. Can. J. Microbiol. 58 (12), 1344-1352. https://doi.org/10.1139/cjm-2012-0468.

Villemant, C., Barbet-Massin, M., Perrard, A., et al., 2011a. Predicting the invasion risk by the alien bee-hawking Yellow-legged hornet Vespa velutina nigrithorax across Europe and other continents with niche models. Biol. Conserv. 144, 2142-2150. https://doi.org/10.1016/j.biocon.2011.04.009.

Villemant, C., Muller, F., Haubois, S., 2011b. Bilan des travaux (MNHN et IRBI) sur l'invasion en France de Vespa velutina, le frelon asiatique prédateur d'abeilles. Proceedings of the Journée Scientifique Apicole-11 February, pp. 3-12.
Visser, A.A., Nobre, T., Currie, C.R., et al., 2012. Exploring the potential for actinobacteria as defensive symbionts in fungus-growing termites. Microb. Ecol. 63 (4), 975-985. https://doi.org/10.1007/s00248-011-9987-4.

Wagner, D.L., 2019. Insect declines in the Anthropocene. Annu. Rev. Entomol. 65 (7), 457-480. https://doi.org/10.1146/annurev-ento-011019-025151 (65).

Wang, Q., Garrity, G.M., Tiedje, J.M., Cole, J.R., 2007. Naïve Bayesian classifier for rapid assignment of rRNA sequences into the new bacterial taxonomy. Appl. Environ. Microbiol. 73 (16), 5261-5267. https://doi.org/10.1128/AEM.00062-07.

Weiss, M., Bauer, R., Sampaio, J.P., Oberwinkler, F., 2014. Tremellomycetes and related groups. Systematics and Evolution. Springer, Berlin, Heidelberg, pp. 331-355.

White, T.J., Bruns, T., Lee, S.J.W.T., Taylor, J., 1990. Amplification and Direct Sequencing of Fungal Ribosomal RNA Genes for Phylogenetics. PCR Protocols: A Guide to Methods and Applications. vol. 18 pp. 315-322.

Wilson, E.O., 1987. Causes of ecological success: the case of the ants. J. Anim. Ecol. 56 (1) 1-9.

Zientz, E., Feldhaar, H., Stoll, S., Gross, R., 2005. Insights into the microbial world associated with ants. Arch. Microbiol. 151 (7), 513-519. https://doi.org/10.1007/s00203005-0041-0. 